\section{Drug-induced lichenoid exanthema by a vaccine against COVID-19 (Vaxzevria)}

\author{
Mathieu Ziraldo, ${ }^{1}$ Ivan Theate, ${ }^{2}$ \\ Olivier Vanhooteghem ${ }^{1}$
}

${ }^{1}$ Dermatology Department, CHU UCL Namur Site de Sainte Elisabeth, Namur; ${ }^{2}$ Institute of Pathology and Genetics, Gosselies, Belgium

\begin{abstract}
A 66-year-old white female presented with a generalized, erythematous, and itchy eruption for 3 days after. She reported having fever on the first day of eruption, complaints of asthenia, and anorexia with no other systemic symptoms. She received her first dose of Vaxzevria (AstraZeneca, Cambridge, UK) against COVID-19 three weeks prior. The eruption started on the right arm at the vaccine injection site and then spread progressively throughout the entire body. We noticed multiform erythema-like patches with three or four concentric circles with different shades of redness. Anatomopathological analysis indicated a lichenoid histological pattern compatible with adverse event of vaccine. Degressive general corticotherapy was prescribed with an improvement of the symptomatology and complete healing in ten days. Physicians should be aware if this rare adverse event. Drug-induced lichenoid exanthema is considered a non-severe reaction and does not contraindicate the readministration of essential drugs. In this case, the patient refused the second injection of Vaxzevria.
\end{abstract}

\section{Introduction}

Drug-induced skin reaction is defined as a cutaneous adverse reaction induced by orally administered drugs. Gell et al. ${ }^{1}$ distinguish nonimmunological and immunological reaction, including four mechanism types for the latter. The diagnosis remains based on clinical, paraclinical and histological criteria. ${ }^{2}$ Drug-induced lichenoid exanthema (DiLE) is an uncommon form of skin drug reaction. Its pathogenesis is not well characterized, and clinical and histological criteria are still essential for diagnosis. Many medications can potentially induce cutaneous reaction, and the number of capable medications increases every year. Rarely, vaccines can be involved in the occurrence of DiLE, such as those against hepatitis B, influenza, herpes zoster, and tetanus-diphtheria-pertussis. ${ }^{3}$ Here, we report, to our knowledge, the first case of DiLE induced by vaccination against coronavirus disease (COVID-19).

\section{Case Report}

A 66-year-old white female presented with a generalized, erythematous and itchy eruption for 3 days. She reported having fever on the first day of eruption, complaints of asthenia, and anorexia with no other systemic symptoms. She had a history of hypertension, which was treated with beta blockers, and breast cancer in remission for 5 years previously, which was treated by tumorectomy and radiotherapy. She has no allergy history. There was no recent change in her usual treatment and no recent infection. She received her first dose of Vaxzevria (AstraZeneca, Cambridge, UK) against COVID-19 three weeks prior. The eruption was erythematous, arranged in rounded patches separated from each other by healthy skin, non-blashkolinear, and dimly symmetrical and did not affect the face or mucous membranes. It started on the right arm at the vaccine injection site and then spread progressively throughout the entire body (Figure 1). We noticed multiform erythema-like patches with three or four concentric circles with different shades of redness. This pattern was particularly pronounced and more irregular at the vaccine injection site on the right arm (Figure 2). Bilateral cervical lymph adenopathy was palpated. Routine laboratory investigation revealed inflammatory syndrome (elevated C-reactive protein levels, hyperferritinaemia, and neutrophilic hyperleukocytosis) and no liver or renal dysfunction. Antinuclear factor, ANCA and hepatitis serology tests were negative. Anatomopathological analysis showed vacuolar alterations of the basal layer with lymphocytic exocytosis without keratinocyte necrolysis and pericapillary lymphocytic infiltrate in the dermis. Immunofluorescence investigations were negative. These findings indicated a lichenoid histological pattern compatible with a cutaneous reaction to vaccine (Figure 3). Patch tests were negative for each component of Vaxzevria. Degressive general corticotherapy was prescribed with an improvement of the symptomatology, clinical signs and laboratory alterations in two days, with complete healing in ten days.
Correspondence: Olivier Vanhooteghem, Dermatology Department, CHU UCL Namur, Site Sainte Elisabeth, Place Louise Godin 15, 5000 Namur, Belgium.

Tel.: +32.495500289.

E-mail: ovanhooteghem@hotmail.com

Key words: Toxidermia, Vaxzevria, Lichenoid, COVID-19, Drug-induced exanthema.

Contributions: The authors contributed equally.

Conflict of interest: The authors declare no potential conflict of interest.

Funding: None.

Ethical approval: Not applicable.

Consent for publication: Written informed consent was obtained from the patient for publication of this case report and any accompanying images. A copy of the written consent is available on request.

Availability of data and material: Data and materials are available by the authors.

Please cite this article as: Ziraldo M, Theate I, Vanhooteghem O. Drug-induced lichenoid exanthema by a vaccine against COVID-19 (Vaxzevria). Dermatol Rep 2021;13:9358.

Received for publication: 23 August 2021. Revision received: 6 September 2021.

Accepted for publication: 12 September 2021

This work is licensed under a Creative Commons Attribution-NonCommercial 4.0 International License (CC BY-NC 4.0).

${ }^{\circ}$ Copyright: the Author(s), 2021

Licensee PAGEPress, Italy

Dermatology Reports 2021; 13:9358

doi:10.4081/dr.2021.9358

\section{Discussion}

The updated French imputability method is commonly used to investigate a drug's causality in the induction of adverse events. ${ }^{4}$ In the era of the severe acute respiratory syndrome coronavirus 2 (SARSCoV-2) pandemic and specific vaccine emergence, studies have been conducted in a shorter time than usual, so adverse events could potentially be underestimated or even not revealed. Furthermore, many vaccines could induce DiLE with variable frequency of occurrence. Allergenic epitopes are suspected for DiLE, such as egg, yeast protein or the vaccine itself. Vaxzevria contains monkey adenoviruses that encode the SARS-CoV-2 spike protein, which is a genetically modified organism; L-histidine; 


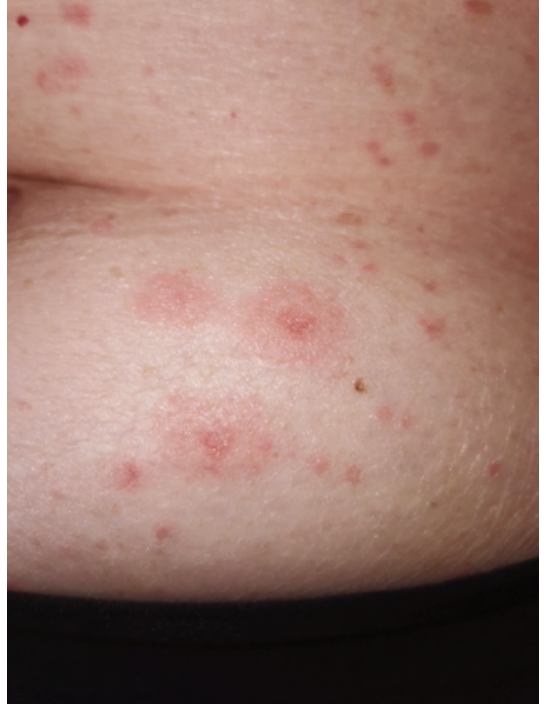

Figure 1. Erythema multiforme like lesions on trunk.

magnesium chloride; polysorbate 80 ; disodium edetate; and water. Aside from the microbiological agent, none of these components is known to induce DiLE. Regarding the second dose, no case of eruption resurgence was described because no reintroduction was performed Nevertheless, similar to other drug-induced reaction, if a lichenoid eruption has occurred with a drug, it is possible that the same reaction will appear more quickly after re-exposure to the same medication or a medication in the same family. ${ }^{5}$ Corticotherapy treatment before and after vaccination could diminish the immunogenicity of the vaccine. ${ }^{6}$

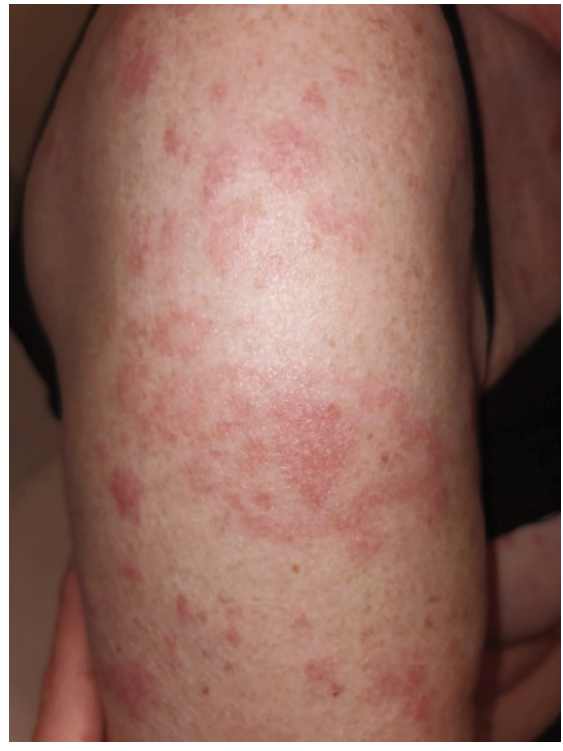

Figure 2. Eruption at injection's site (right arm).

\section{Conclusions}

In this case report, intrinsic imputability is plausible, but extrinsic imputability is undefined due to the recent marketing authorization and lack of scientific publications for COVID-19 vaccines. Therefore, semiological and chronological signs support a plausible risk of lichenoid exanthema with the Vaxzevria vaccine. Physicians should be aware if this rare adverse event. DiLE is considered a non-severe druginduced cutaneous reaction and does not contraindicate the re-administration of essential drugs. ${ }^{5}$ In this case, the patient refused the second injection of Vaxzevria.

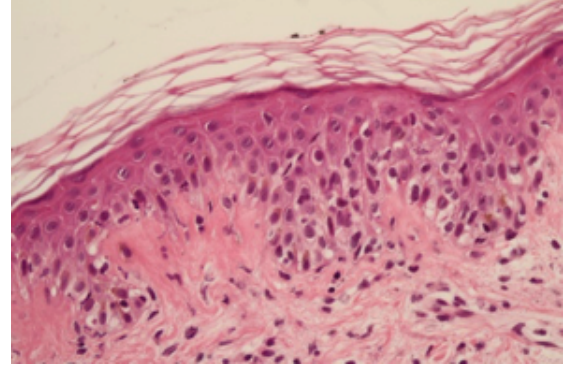

Figure 3. Lichenoid alteration of the basal layer (HE 25x).

\section{References}

1. Gell PGH, Coombs RRA. Clinical aspects of immunology. Blackwell Sci Publ 1963;1-1386.

2. Lai YC, Yew YW. Lichen planus and lichenoid drug eruption after vaccination. Cutis 2017;100:E6-20.

3. Virath R, Balai M, Gupta LK. Blachkoid lichenoid drug eruption due to tenofovir. Indian Dermatol Online J 2020;11:826-7.

4. Bégaud B, Evreux JC, Jouglard J, Lagier G. [Imputation of the unexpected or toxic effects of drugs. Actualization of the method used in France]. Therapie 1985;40:111-8. [In French].

5. Bourrain JL. Cutaneous drug reactions. Ann Dermatol Vénéréol 2019;146:74055.

6. Zimmermann P, Curtis N. Factors that influence the immune response to vaccination. Clin Microbiol Rev 2019;32:e0084-18. 